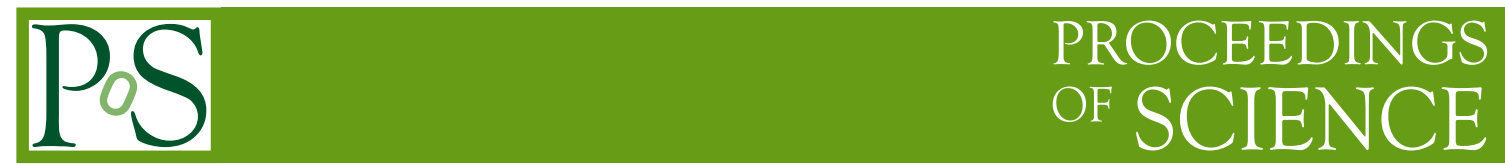

\title{
Theory overview of heavy flavour production
}

\author{
Eric Laenen* \\ ITFA, University of Amsterdam, Science Park 904, 1090 GL Amsterdam, \\ ITF, Utrecht University, Leuvenlaan 4, 3584 CE Utrecht \\ Nikhef, Science Park 105, 1098 XG Amsterdam, The Netherlands \\ E-mail: Eric.Laenen@nikhef.nl
}

A short overview of the present understanding of heavy flavour hadroproduction is given. Auxiliary issues such as the definition of the heavy flavour mass and of a heavy flavour parton density are touched upon.

The 13th International Conference on B-Physics at Hadron Machines - Beauty2011, April 04-08, 2011

Amsterdam, The Netherlands

${ }^{*}$ Speaker. 


\section{Introduction}

The main topic at this conference being the study of heavy flavoured hadron decays, one may to first approximation only care that heavy flavours are produced in sufficient numbers - as indeed happens at B-factories, the Tevatron and the LHC - and leave the detailed understanding of production dynamics to other conferences. Nevertheless, because for a number of studies one cannot fully decouple production from decay, and for general background knowledge, a brief review of our present understanding of heavy flavour hadroproduction may be useful.

Heavy quarks have a number of distinguishing properties as far as the description of their production goes. First, because their masses well exceed $\Lambda_{Q C D}$, a perturbative description is natural as the mass sets a hard scale. It also creates a threshold, and thereby possibly interesting dynamics. Second, heavy quark taggability, through semi-leptonic decay for instance, allows a calculation per individual flavour, which in turn can yield information about certain parton distribution functions (PDFs). These properties enable quite detailed and precise understanding of heavy flavour production in hadron colliders. In what follows I discuss these and other issues in somewhat more detail. However, due to limited space and broad scope the list of references cited is far from complete.

\section{Heavy flavour production at NLO and beyond}

By and large, the state of the art in exact calculations for heavy flavour production is nextto-leading order (NLO) accuracy, possibly matched to parton shower simulations. The benefits of going beyond leading order (LO) are well-known: production rates are more accurately predicted due to reduced scale dependence, and distributions are more realistically modelled because of the extra parton. Moreover, by their size, the corrections self-diagnose the perturbative approach as such for the observable at hand. A general review of the benefits of perturbation theory at colliders can be found in Ref. [1].

To facilitate later discussions, let us briefly review the essential aspects of a NLO calculation. A LO calculation consists of those diagrams that contain the desired final state with a mininum of extra particles. The NLO approximation consists virtual corrections to those, as well as diagrams having one extra radiated parton, over whose phase space one integrates over, either wholly or partially. These loop momentum and phase space integrations produce, besides finite terms, regularized infinities of UV or IR nature. The former are straightforwardly handled using standard renormalization methods. The latter cancel between virtual and real graphs, not just for the inclusive cross section, but also when the momentum of the extra emitted parton is kept available, i.e. for fully differential cross sections. How to do this is not entirely straightforward, nor unique, and can be done by phase space slicing, or using "subtraction schemes" such as antenna, dipole, and FKS subtractions. In recent years, innovative methods have lead to a veritable revolution in the amount and complexity of NLO calculations, many with heavy flavours, and are rapidly approaching full automatization, including parton showers. Beyond NLO no full NNLO calculation exists as yet for heavy flavour hadroproduction, though much progress is made, see [2] for a recent presentation of the status. A large set of approximate calculations based on threshold resummation exists, primarily for fully or single-particle inclusive cross sections. 


\section{Heavy flavour PDF's}

It is a subtle issue to choose a description for heavy flavour production in collisions with initial hadrons, such as deep-inelastic scattering or hadroproduction. One must decide whether one wants to employ a fixed number of light flavours in the evolution of the parton densities and $\alpha_{s}$ and treat the heavy flavour as an external quantum field: the "fixed flavour-number scheme" (FFNS); or whether one changes the number of dynamical flavours depending on the scale present in the parton subprocess, and then introduce heavy quark PDFs: the "variable flavour-number scheme" (VFNS). The latter comes in two general varieties: (i) the Zero Mass VFNS, where the mass dependence only comes in through the matching point $\mu=m$, where the number of active flavors in the evolution of the PDF's changes by one, effectively summing $\ln (Q / m)$ to all orders, and (ii) the General Mass VFNS, where the mass is also kept in the partonic coefficient functions and gives a better approximation in the near-threshold region. In so doing one may control $\ln (Q / m)$ logarithms to all orders, without relinquishing control over the region $Q \simeq m$. The past few years have seen much progress in the development of such schemes, which began in [3], to NLO and NNLO. This renewed attention is spurred in particular by the realization that different realizations of VFNS schemes led to variations of up to $10 \%$ in key LHC cross sections. For a concise review of which PDF group uses which method to deal with heavy flavors, see [4] , and references therein. Similar issues occur in the final state in heavy flavor production at high $p_{T}$, where one might wish to control powers of $\ln \left(p_{T} / m\right)$ to all orders. A variant of GM-VFNS, called FONLL [5] was developed, and its importance demonstrated in the correct explanation of the Tevatron $b$-quark $p_{T}$ distribution [6].

\section{Charm and bottom}

There are many aspects in common between charm and bottom production at the Tevatron or LHC. One, just discussed, is that their masses, though considerably larger than $\Lambda_{Q C D}$, are mostly sufficiently small compared to the typical hard scale to consider them in a sense as partons. Differences can matter though: $W+$ charm production is directly sensitive to the strange quark PDF, but $W+$ bottom is less directly connected to a particular PDF. Indeed, their production in association with jets is a major component of the signal and background to new physics searches, and an impressive array of NLO calculations for them are now available, see [7] for a recent presentation of the status.

\subsection{Onia}

Thus far we have only discussed open heavy flavour production, but the production of their bound states is a very interesting area in its own right, involving new dynamics and different descriptions in terms of effective field theory.

Non-relativistic QCD (NRQCD) [8] is the effective field theory for light quarks, gluons and heavy quark-antiquark bound states (onia). This effective theory allows, besides an expansion in $\alpha_{s}$, a systematic expansion in $v$, the heavy quark velocity inside the onium bound state. NRQCD factorizes the physics of production, involving the heavy flavour mass $m$, and a hard scale such 
as $p_{T}$, from the physics of binding, associated with the inverse onium size $m v$ and binding energy $m v^{2}$, where $v^{2} \simeq 0.3,0.1$ for $J / \psi$ and $\Upsilon$, resp.

Measurements at the Tevatron and at HERA left a puzzle in high- $p_{T}$ onium production. HERA measurements are in reasonable agreement with the straightforward color-singlet model, in which one computes the $Q \bar{Q}$ in a color singlet state at zero relative momentum, and subsequently projects onto the appropiate spin- and angular momentum quantum numbers. However, a Tevatron measurement [9] of the production rates of the $J / \psi, \psi^{\prime}$ states revealed a large excess over the prediction of the colour-singlet model [10]. In the NRQCD framework [11] onium hadroproduction is described by

$$
d \sigma(p+\bar{p} \rightarrow \psi(P, \lambda)+X)=\sum_{i j} f_{i} \otimes f_{j} \otimes \sum_{n} d \sigma(i+j \rightarrow Q \bar{Q}[n]+X)\left\langle O_{n}^{\psi(\lambda)}\right\rangle
$$

where the sum is over all allowed states, labeled by the spectroscopic notation ${ }^{2 S+1} L_{J}$, possibly in a non-singlet color state, as indicated by the colour label $(n=\mathbf{1}, \mathbf{8}, \ldots)$. The operators $O_{n}^{\psi(\lambda)}$ encode the binding physics, and are ordered in the expansion parameters $\alpha_{s}$ and $v$. The fact that coloured channels are open to scatter into, especially the gluon fragmentation mechanism, leads to a large increase in the cross section [11], providing an explanation for the Tevatron data.

A test of the importance of these colour-octet states is possibly the polarization of the vector onium octet-state produced, $J / \psi$ and $\psi^{\prime}[12,13,14]$. A heuristic argument says that at large $p_{T} / m$, this state results in essence from the fragmentation of a nearly on-shell and transverse gluon, leading to a transversely polarized onium state, as soft-gluon binding effects do not flip the spin. Especially the $\Upsilon$ polarization, having smaller $v$, should conform to this expectation, but the most recent $\mathrm{CDF}$ and D0 measurements $[15,16]$ leave the situation somewhat unclear, indicating at least an important contribution of longitudinally polarized onia. There has been considerable recent progress at the theoretical level (see [17] for a recent overview), such as the NLO corrections to ${ }^{3} S_{1}$ color singlet production of onia $[18,19]$, the available of automatic computation of tree-level quarkonium processes [20], and a promising factorized formalism beyond leading power [21]. With this progress in hand, one may look forward to LHC experiments soon weighing with high-statistics polarization measurements at high $p_{T}$.

\section{Heavy flavour mass}

Since heavy flavours cannot propagate over macroscopic distances, in contrast to electrons e.g., there is an intrinsic difficulty in defining the heavy quark mass through the pole of the full propagator. Though a natural definition, and a requirement for the definition of a particle mass in scattering amplitudes - such a pole only exists in perturbation theory, and event then its location is intrinsically ambiguous by $\mathscr{O}\left(\Lambda_{Q C D}\right)[22,23,24]$. A theoretically more precise definition is the $\overline{M S}$ mass $\bar{m}(\mu)$ whose relation to the pole mass is known to sufficiently high order. For $\mu$ one often takes the implicit value found when intersecting the $\bar{m}(\mu)$ curve with the $\bar{m}(\mu)=\mu$ axis, yielding $\bar{m}(\bar{m})$.

For the top quark, this value is about $10 \mathrm{GeV}$ smaller than the pole mass, and thus the question often arises what mass the Tevatron and LHC experiments measure [25]. Experimentally, the top quark mass is reconstructed by collecting jets and leptons. Soft particles arising from both within 
and outside these jets may affect the reconstructed mass. Moreover, various experimental methods used (e.g. track quality cuts), and Monte Carlo based corrections do not have a clean perturbation theory description. The question is difficult to answer, but the pole mass seems close to the true answer.

\section{Top}

The top quark is an especially interesting heavy quark, because its large top mass implies that it couples strongly to whatever breaks the electroweak symmetry, and that its resulting large width minimizes obscuring hadronization effects and allows preservation of spin information. Top is one the hand a troublemaker for the Standard Model, contributing significantly to the quadratic divergences of the Higgs self energy, but is at the same time an life raft for beyond the Standard Model (BSM) theories such as the MSSM, raising the upper limit on the light Higgs in that theory. An extensive review of top physics is beyond our scope, so let us focus on a few salient features.

An important top quark property is of course its mass. The Tevatron experiments have set the standard to a level that will be hard to pass by the LHC by measuring it to about half a percent accuracy $(173.2 \pm 0.9 \mathrm{GeV})$. Together with an accurately measured $W$ boson mass it severely constrains the mass range of a possible Higgs boson both in the Standard Model, or for that matter in the MSSM. Note, however, to constrain the Standard Model parameters $m_{W}$ and $m_{H}$ one does not need much more accuracy than that, though a careful mass definition and extraction would be welcome. To constrain new physics, once discovered, a much more precisely determined top mass, only achieveable at a linear colliders, would be required.

Another key measurement involves the pair production cross section. The latest theoretical predictions differ in their particular implementations of threshold resummation. When the top quark pair is produced near threshold, logarithms whose arguments represent the distance to threshold in the perturbative series become numerically large. It is important to note here that the definition of the threshold can depend on the observable. Thus, for the inclusive cross section threshold is given by $T_{1}: s-4 m^{2}=0$. For the transverse momentum distribution we have $T_{2}: s-4\left(m^{2}+p_{T}^{2}\right)=0$, and for the doubly differential distribution in $p_{T}$ and rapidity we have $T_{3}: s-4\left(m^{2}+p_{T}^{2}\right) \cosh y=0$. The perturbative series for any of these (differential) cross sections can be expressed as

$$
d \sigma\left(T_{\alpha}\right)=\sum_{n} \sum_{k}^{2 n} \alpha_{s}^{n} c_{n, k}^{\alpha} \ln ^{k}\left(T_{\alpha}\right)
$$

plus non-logarithmic terms. Note that it is allowed to use e.g. $T_{2}$ for the inclusive cross section, by first analyzing $d \sigma / d p_{T}$ and then integrating over $p_{T}$. For any complete fixed order calculation this will give the same answer, but if one only knows the logarithmic terms because the exact answer is unknown, numerical differences will occur which constitute a theoretical uncertainty [26].

The charge asymmetry is the difference in production rate for top and anti-top at fixed angle or rapidity. While electroweak production via a $Z$-boson could produce a (very small) asymmetry at LO, QCD itself does produce it at $\mathscr{O}\left(\alpha_{s}^{3}\right)$ through a term proportional to the $\mathrm{SU}(3) d_{a b c}$ symbol [27, 28, 29, 30, 31]. Recent CDF [32] and [33] measurements are considerably larger than the Standard Model predictions. Recently it was shown that when including even higher order corrections, which at this stage can only be assessed from approximate, resummation based calculations [26, 34], and 
was studied in Ref. [35, 36]. At the LHC, where the $g g$ channel dominates, the asymmetry is naturally small, but may be enhanced at large invariant mass, where the $q \bar{q}$ channel regains strength. The charge asymmetry is present at leading order in $t \bar{t}+$ jet production. However, NLO corrections [30] appear to wash out the asymmetry for this reaction.

\section{Conclusions}

This brief overview is intended to recall the key notions associated wth heavy flavour production at hadron colliders, and their importance. The overall picture emerging is that for nearly all ways of producing heavy flavours, major theoretical progress, often helpfully encoded in public codes, is met by impressive new data of Tevatron and especially LHC, leading to much enhanced understanding of the precise mechanisms of production. This should benefit producers and consumers of heavy flavours alike.

\section{References}

[1] Eric Laenen and Doreen Wackeroth. Radiative corrections for the LHC and linear collider era. Ann.Rev.Nucl.Part.Sci., 59:367-396, 2009.

[2] P. Uwer. Top physics. contribution to QCD at the LHC, St. Andrews, Scotland, Aug. 22-26, 2011.

[3] M. A. G. Aivazis, John C. Collins, Fredrick I. Olness, and Wu-Ki Tung. Leptoproduction of heavy quarks. 2. a unified qcd formulation of charged and neutral current processes from fixed target to collider energies. Phys. Rev., D50:3102-3118, 1994.

[4] A. De Roeck and R.S. Thorne. Structure Functions. 2011. * Temporary entry *.

[5] Matteo Cacciari, Mario Greco, and Paolo Nason. The $\mathrm{p}(\mathrm{t})$ spectrum in heavy flavor hadroproduction. JHEP, 05:007, 1998.

[6] Matteo Cacciari and Paolo Nason. Is there a significant excess in bottom hadroproduction at the tevatron? Phys. Rev. Lett., 89:122003, 2002.

[7] G. Zanderighi. Gauge boson production at colliders. contribution to Lepton Photon 2011, Mumbai, India, Aug. 22-27, 2011.

[8] Geoffrey T. Bodwin, Eric Braaten, and G. Peter Lepage. Rigorous qcd analysis of inclusive annihilation and production of heavy quarkonium. Phys. Rev., D51:1125-1171, 1995.

[9] F. Abe et al. Inclusive $\mathrm{j} / \mathrm{psi}$, psi (2s) and $\mathrm{b}$ quark production in anti- $\mathrm{p} \mathrm{p}$ collisions at $\mathrm{s}^{* *}(1 / 2)=$ 1.8-tev. Phys. Rev. Lett., 69:3704-3708, 1992.

[10] Gerhard A. Schuler. Quarkonium production and decays. 1994.

[11] Eric Braaten and Sean Fleming. Color octet fragmentation and the psi-prime surplus at the tevatron. Phys. Rev. Lett., 74:3327-3330, 1995.

[12] Peter Cho and Mark B. Wise. Spin symmetry predictions for heavy quarkonia alignment. Phys. Lett., B346:129-136, 1995.

[13] Peter Cho, Mark B. Wise, and Sandip P. Trivedi. Gluon fragmentation into polarized charmonium. Phys. Rev., D51:2039-2043, 1995. 
[14] M. Beneke and I. Z. Rothstein. Psi-prime polarization as a test of color octet quarkonium production. Phys. Lett., B372:157-164, 1996.

[15] Thomas Kuhr. Upsilon polarization measurement at CDF. PoS, DIS2010:159, 2010.

[16] V.M. Abazov et al. Measurement of the polarization of the $v_{1 S}$ and $v_{2 S}$ states in $p \bar{p}$ collisions at $\sqrt{s}=$ 1.96-TeV. Phys.Rev.Lett., 101:182004, 2008.

[17] N. Brambilla et al. Heavy quarkonium: progress, puzzles, and opportunities. Eur. Phys. J., C71:1534, 2011.

[18] John M. Campbell, F. Maltoni, and F. Tramontano. QCD corrections to J/psi and Upsilon production at hadron colliders. Phys. Rev. Lett., 98:252002, 2007.

[19] Bin Gong and Jian-Xiong Wang. Next-to-leading-order QCD corrections to J/psi polarization at Tevatron and Large-Hadron-Collider energies. Phys. Rev. Lett., 100:232001, 2008.

[20] Pierre Artoisenet, Fabio Maltoni, and Tim Stelzer. Automatic generation of quarkonium amplitudes in NRQCD. JHEP, 0802:102, 2008.

[21] Zhong-Bo Kang, Jian-Wei Qiu, and George Sterman. Factorization and quarkonium production. Nucl. Phys. Proc. Suppl., 214:39-43, 2011.

[22] M. Beneke and V. M. Braun. Heavy quark effective theory beyond perturbation theory: Renormalons, the pole mass and the residual mass term. Nucl. Phys., B426:301-343, 1994.

[23] I. I. Bigi, M. A. Shifman, N. G. Uraltsev, and A. I. Vainshtein. The pole mass of the heavy quark. perturbation theory and beyond. Phys. Rev., D50:2234-2246, 1994.

[24] Martin C. Smith and Scott S. Willenbrock. Top quark pole mass. Phys. Rev. Lett., 79:3825-3828, 1997.

[25] Andre H. Hoang and Iain W. Stewart. Top Mass Measurements from Jets and the Tevatron Top-Quark Mass. Nucl.Phys.Proc.Suppl., 185:220-226, 2008.

[26] Nikolaos Kidonakis, Eric Laenen, Sven Moch, and Ramona Vogt. Sudakov resummation and finite order expansions of heavy quark hadroproduction cross sections. Phys. Rev., D64:114001, 2001.

[27] P. Nason, S. Dawson, and R. K. Ellis. The one particle inclusive differential cross-section for heavy quark production in hadronic collisions. Nucl. Phys., B327:49-92, 1989.

[28] W. Beenakker, W. L. van Neerven, R. Meng, G. A. Schuler, and J. Smith. Qcd corrections to heavy quark production in hadron-hadron collisions. Nucl. Phys., B351:507-560, 1991.

[29] Johann H. Kuhn and German Rodrigo. Charge asymmetry of heavy quarks at hadron colliders. Phys.Rev., D59:054017, 1999.

[30] S. Dittmaier, P. Uwer, and S. Weinzierl. NLO QCD corrections to $t$ anti-t + jet production at hadron colliders. Phys. Rev. Lett., 98:262002, 2007.

[31] Wolfgang Hollik and Davide Pagani. The electroweak contribution to the top quark forward-backward asymmetry at the Tevatron. 2011. * Temporary entry*.

[32] T. Aaltonen et al. Evidence for a Mass Dependent Forward-Backward Asymmetry in Top Quark Pair Production. Phys. Rev., D83:112003, 2011.

[33] V.M. Abazov et al. First measurement of the forward-backward charge asymmetry in top quark pair production. Phys.Rev.Lett., 100:142002, 2008. 
[34] Valentin Ahrens, Andrea Ferroglia, Ben D. Pecjak, and Li Lin Yang. Precision predictions for the $\mathrm{t}+\mathrm{t}$ (bar) production cross section at hadron colliders. Phys. Lett., B703:135-141, 2011.

[35] Leandro G. Almeida, George F. Sterman, and Werner Vogelsang. Threshold Resummation for the Top Quark Charge Asymmetry. Phys.Rev., D78:014008, 2008.

[36] V. Ahrens, A. Ferroglia, M. Neubert, B. D. Pecjak, and L. L. Yang. RG-improved single-particle inclusive cross sections and forward-backward asymmetry in $t \bar{t}$ production at hadron colliders. 2011. 